\title{
RECHERCHE D'UNE TRANSMISSION ÉVENTUELLE DES VIRUS SHV ET NHI AUX POPULATIONS NATURELLES A PARTIR DE SALMONICULTURES CONTAMINÉES.
}

\author{
P. GIRAUD (1), J. FACON (2), J. CATEL (1), H. KNOCKAERT (2), \\ J. GUENOT (1), J.M. SEVIN (1)
}

(1) Laboratoire Vétérinaire Départemental du Pas-de-Calais, SP 18, 62022 ARRAS Cedex.

(2) Direction des Services Vétérinaires du Pas-de-Calais, SP 19, 62022 ARRAS Cedex.

Reçu le 05 mars 1993

Accepté le 31 mars 1994
Receveid 05 March, 1993 Accepted 31 March, 1994

\section{RÉSUMÉ}

L'étude s'est déroulée sur une portion de $25 \mathrm{Km}$ de rivière de première catégorie du Pas-de-Calais, le long de laquelle se trouvent 4 salmonicultures contaminées par les virus SHV et/ou NHI. Des pêches électriques ont été effectuées en juin, septembre et octobre 1991 au niveau de 4 sites choisis en amont et en aval des salmonicultures. Elles ont permis de prélever un échantillonnage représentatif de poissons vivants autres que salmonidés pour une recherche virologique ( 85 analyses par lot de 3 ou 5 sujets) et de réaliser 96 sérologies SHV et NHI sur des prises de sang de truites capturées en rivière. Une soixantaine de sérologies ont également été faites dans chacune des piscicultures en décembre 1991. Au niveau de la rivière, toutes les virologies et les sérologies sont négatives. Au vu de ces résultats, le niveau de contamination et de portage de rhabdovirus dans le milieu naturel en relation avec des salmonicultures infectées semble relativement bas, ce qui n'exclut pas la possibilité de transmission par le biais des poissons sauvages.

\section{STUDY OF A POSSIBLE TRANSMISSION OF VHS AND IHN VIRUSES FROM INFECTED TROUT FARMS TO WILD FISH POPULATIONS.}

\section{SUMMARY}

The study took place on a portion of first category river of the Pas-de-Calais, along which exist 4 trout farms infected by the virus VHS and/or IHN. Electric fishing was carried out in June, September and October 1991 on 4 stretches up and downstream of the trout farms. This allowed a representative sampling of live fish other than salmonidae for virus isolation ( 85 analyses by batch of 3 or 5 individuals) and to realize 96 serological tests for VHS and IHN on blood samples of trouts captured in the river. About sixty serological tests were also made in each of the 4 fish farms in December 1991. As far as the river is concerned, all the virus isolations and the serological tests were negative. Considering these results, the level of contamination and carriage of rhabdoviruses in the natural milieu connected with infected trout farms seems relatively low, but this does not rule out the possibility of transmission by wild fish. 


\section{INTRODUCTION}

La septicémie hémorragique virale (SHV)(JENSEN, 1965), présente en France depuis de nombreuses années (BELLET, 1954) et la nécrose hématopoïétique infectieuse (NHI)(AMEND et al., 1969), d'implantation plus récente (DE KINKELIN et al., 1987) sont deux rhabdoviroses contagieuses à l'origine de maladies aiguës chez de nombreux salmonidés, principalement la truite arc-en-ciel dans les eaux continentales, causant d'importantes pertes dans les salmonicultures. De nombreux travaux expérimentaux ont porté sur la réceptivité de différents salmonidés et d'autres espèces de poissons à ces deux rhabdovirus: sensibilité des corégones (Coregonus sp.) au virus de la SHV (AHNE et THOMSEN, 1985), du brochet (Esox lucius) à celui de la SHV et de la NHI (DORSON et al., 1987), non sensibilité du gardon (Leuciscus rutilus), du chevaine (Leuciscus cephalus) (TACK, 1959), de la tanche (Tinca vulgaris) (ZWILLENBERG et al., 1968). Cependant peu d'études ont été menées dans le milieu naturel pour mettre en évidence ces deux virus dans des populations pisciaires sauvages. L'objectif de notre étude est la recherche d'une transmission éventuelle de ces deux virus aux poissons sauvages à partir de salmonicultures contaminées.

\section{MATÉRIELS ET MÉTHODES}

\section{Prélèvements}

Le site retenu est une rivière de première catégorie du Pas-de-Calais sur laquelle sont situées quatre salmonicultures (figure 1). En 1990, le virus de la NHI a été mis en évidence dans la pisciculture D, le virus SHV dans la pisciculture $\mathrm{C}$; dans ces deux piscicultures, des sérologies effectuées en 1990 ont été positives pour la NHI. Les 3 piscicultures les plus en amont appartiennent au même propriétaire et présentent des statuts sanitaires équivalents d'après la Direction des Services Vétérinaires du Pas-deCalais. Aucune action d'assainissement n'a été entreprise dans ces 4 piscicultures avant notre étude.

De septembre à octobre 1991, une série de 8 pêches électriques réparties sur la rivière (figure 1) ont été effectuées dans quatre sites en amont et en aval des principales piscicultures et ont permis :

- de faire des prises de sang sur les truites sauvages pour une sérologie SHV et NHI,

- de capturer et d'amener au laboratoire des spécimens des principales espèces rencontrées autres que celles des salmonidés pour une recherche virologique; le choix s'est porté sur les espèces les plus représentées dans le biotope, représentation estimée lors d'un sondage préparatoire effectué en juin 1991 (figure 1) et sur les espèces servant de nourriture aux truites.

En décembre 1991, un sondage sérologique pour la SHV et la NHI a été réalisé dans 3 salmonicultures avec en moyenne une soixantaine d'échantillons par pisciculture.

\section{Virologie : (DE KINKELIN et al., 1985)}

Les lignées cellulaires utilisées, cellules EPC (Epithelioma papulosum cyprini) (FIJAN et al., 1983) et cellules RTG2 (Rainbow Trout Gonad) (WOLF et QUIMBY, 1962), étaient cultivées en milieu de Eagle Tris modifié Glasgow pH 7,45, additionné de phosphate de Tryptose $(10 \%)$, de sérum d'embryon de bovin (10\% croissance - $2 \%$ entretien) et d'antibiotiques (pénicilline, streptomycine, kanamycine).

La technique utilisée était celle du laboratoire de référence Centre National d'Etude Vétérinaire et Alimentaire - Laboratoire Central de Recherche Vétérinaire de Maisons-Alfort -Service d'Ichtyopathologie (CNEVA-LCRV).

Les poissons étaient euthanasiés et traités par lot de 3 ou 5 suivant la taille. Un broyat d'encéphale, de rate et de rein antérieur était repris avec du milieu Earle additionné d'antibiotiques (dilution au $1 / 10^{\circ}$ environ) et centrifugé à $3000 \mathrm{~g}$ pendant $10 \mathrm{mn}$ à $+4{ }^{\circ} \mathrm{C}$, $0,1 \mathrm{ml}$ du surnageant pur et dilué au $1 / 10^{\mathrm{e}}$ était inoculé sur un tapis cellulaire de $24 \mathrm{~h}$ des 


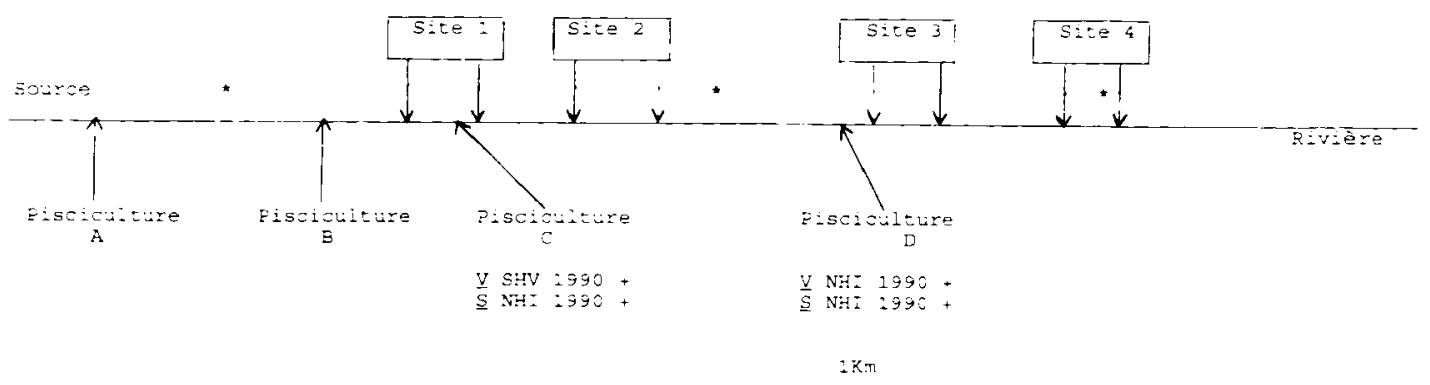

Figure 1 : Diagramme représentant les lieux de prélèvements sur une rivière de première catégorie du Pas-de-Calais.

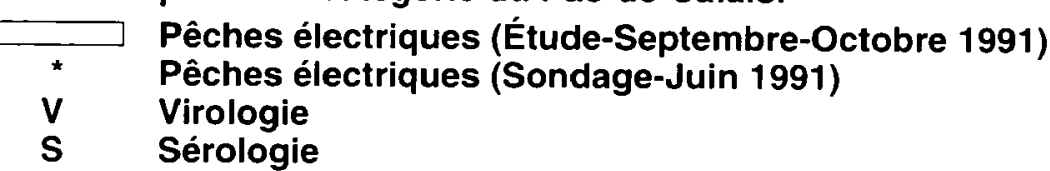

Figure 1 : Diagram showing the sites of the samplings on a first category river of Pasde-Calais.

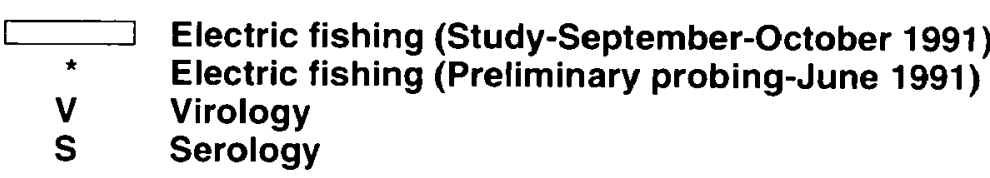

deux lignées EPC et RTG (2 cupules par dilution et par lignée sur une plaque de 24 cupules Nunc). Si aucun effet cytopathogène n'apparaissait après 7 jours de culture, un deuxième passage à partir du surnageant de la première culture était réalisé dans les mêmes conditions. En l'absence d'effet cytopathogène, un troisième et dernier passage était effectué.

Les virus isolés non séroneutralisés par des anticorps de lapin anti NPI étaient envoyés au CNEVA-LCRV de Maisons-Alfort, pour identification (le sérotypage des souches n'a pas été effectué).

\section{Sérologie}

Le sang était prélevé sur place lors des pêches électriques et apporté le jour même au laboratoire. Les tubes de sang étaient alors placés une nuit à $+4^{\circ} \mathrm{C}$ puis centrifugés à $3000 \mathrm{~g}$ pendant $10 \mathrm{mn}$ à $+4{ }^{\circ} \mathrm{C}$. Le sérum était ensuite récupéré stérilement et décomplémenté $30 \mathrm{mn}$ à $45^{\circ} \mathrm{C}$ au bain-marie.

Les tests sérologiques ont été pratiqués suivant la microméthode en plaques du laboratoire de référence CNEVA-LCRV de Maisons-Alfort (RNE Pr. 109/00 IS 410/00 et IS 290/00, 1993).

Le titrage des anticorps spécifiques de la NHI et de la SHV a été réalisé par séroneutralisation sur cellules EPC avec virus fixe et sérum variable. Les dilutions des sérums sont effectuées de 2 en 2 sous un volume de $25 \mu \mathrm{l}$ en milieu de Eagle et sont mises en présence de $25 \mu \mathrm{l}$ de suspension virale avec DEAE et sérum normal de truite préalablement titré (la dilution virale correspond à la plus petite quantité de virus détruisant à $95 \%$ le tapis cellulaire). Les microplaques à 96 puits (Nunc) étaient incubées une nuit à $+4{ }^{\circ} \mathrm{C}$. 
On ajoutait ensuite dans chaque cupule $150 \mu \mathrm{l}$ de suspension cellulaire ajustée à $2,5 \times 10^{6}$ cellules $/ \mathrm{ml}$ dans du milieu Eagle à $5 \%$ sérum de veau foetal et à $0,05 \mathrm{mg} / \mathrm{ml}$ de DEAE.

Les plaques étaient incubées à $+14^{\circ} \mathrm{C}$ et examinées régulièrement. L'incubation à $14{ }^{\circ} \mathrm{C}$ durait de 4 à 6 jours pour la sérologie NHI et de 3 à 5 jours pour la sérologie SHV. Les plaques étaient fixées et colorées au cristal violet à $0,26 \%$ quand le sérum de titre connu atteignait le titre souhaité.

L'activité neutralisante du sérum correspond à la dilution la plus élevée qui inhibe l'installation de $95 \%$ de lésions cellulaires. Le sérum est considéré comme positif à partir du $1 / 64^{e}$ pour la NHI (HATTENBERGER et al., 1989) et du $1 / 32^{\circ}$ pour la SHV (BERNARD et al., 1985). Pour les positifs, la dilution maximale protectrice est indiquée. La sérologie SHV détecte les anticorps contre les trois sérotypes SHV I, SHV II et SHV III.

\section{RÉSULTATS}

Le Tableau I regroupe l'ensemble des 85 résultats de virologie de l'étude qui sont tous négatifs. En complément, 4 lots de 3 lamproies (Lampetra sp.) prélevées sur le site 6 en avril 1992, période où cette espèce était présente, ont été également analysés selon la même méthode et sont négatifs.

Sur les 96 sérums de truites de plus de $250 \mathrm{~g}$ capturées en rivière comprenant $20 \%$ de truites arc-en-ciel (Oncorhynchus mykiss) et $80 \%$ de truites fario (Salmo trutta fario), 31 provenaient des pêches électriques effectuées lors du sondage préparatoire en juin 1991. A l'exception de 2 sérologies ininterprétables, les 94 restantes sont négatives vis-à-vis des deux virus.

Dans les salmonicultures (Tableau II), 173 sérums ont été analysés (60 en moyenne par pisciculture). Des sérums positifs pour la SHV et la NHI ont été trouvés dans les deux piscicultures les plus en aval et essentiellement sur des truites arc-en-ciel. La pisciculture située le plus en amont sur la rivière n'a pas fait l'objet de prélèvements. Le dernier sondage sérologique dans cet établissement remontait à février 1990 ; les 30 sérologies $\mathrm{NHI}$ étaient alors négatives.

Une sérologie NPI a été également réalisée sur les 31 sérums prélevés lors du sondage préparatoire. Elle était négative.

\section{DISCUSSION}

\section{Echantillonnage}

En fonction de l'époque et du site de la pêche électrique, la représentation des différentes espèces est variable. Des fluctuations importantes de la population de chabots, de vairons et de goujons dans une moindre mesure, ont été remarquées et ont quelque peu gêné l'échantillonnage prévu au départ de l'opération. Ceci explique le nombre variable de lots par espèce et l'absence de brochet (Esox lucius) et de brème (Abramis brama) pourtant capturés lors de sondages antérieurs.

\section{Epoque}

Pour des raisons pratiques et de disponibilités de différents intervenants, l'étude n'a pas pu être décalée vers des mois plus froids, plus propices à l'expression clinique de rhabdoviroses et donc à une contamination plus importante du milieu qu'en période d'infection subclinique ou de portage.

\section{Sérologie dans les Salmonicultures}

Les deux piscicultures les plus en aval sur la rivière ne sont pas indemnes de SHV et de NHI au vu des résultats sérologiques. II est donc probable que ces établissements excrétent ou ont excrété des virus SHV etou NHI dans la rivière et contaminent ou ont contaminé éventuellement des espèces réceptives. 
Tableau I : Résultats des analyses virologiques (Septembre-Octobre 1991). Nég : Négatif

Table I : Results of virological analyses (September-October 1991). Neg : Negative

\begin{tabular}{|c|c|c|c|c|c|c|c|c|c|c|}
\hline \multirow{3}{*}{ ESPE்CES } & \multirow{3}{*}{$\begin{array}{l}\text { Nombre } \\
\text { de lots }\end{array}$} & \multirow{3}{*}{$\begin{array}{l}\text { Nbre din- } \\
\text { dividus }\end{array}$} & \multicolumn{8}{|c|}{ SITE DE PRÉLEVEMENT } \\
\hline & & & \multicolumn{2}{|c|}{ Site1 } & \multicolumn{2}{|c|}{ Site 2} & \multicolumn{2}{|c|}{ Site 3} & \multicolumn{2}{|c|}{ Site 4} \\
\hline & & & $\begin{array}{c}\text { Nbre de } \\
\text { Lots }\end{array}$ & $\begin{array}{l}\text { Viro- } \\
\text { logie }\end{array}$ & $\begin{array}{c}\text { Nbre de } \\
\text { Lots }\end{array}$ & $\begin{array}{l}\text { Viro- } \\
\text { logie }\end{array}$ & $\begin{array}{l}\text { Nbre de } \\
\text { Lot }\end{array}$ & $\begin{array}{l}\text { Viro- } \\
\text { logie }\end{array}$ & $\begin{array}{l}\text { Nbre de } \\
\text { Lots }\end{array}$ & $\begin{array}{l}\text { Viro- } \\
\text { logie }\end{array}$ \\
\hline $\begin{array}{l}\text { Anguille } \\
\text { (Anguilla } \\
\text { anguilla) }\end{array}$ & 24 & 72 & & & 14 & Nég & & & 10 & Nég \\
\hline $\begin{array}{l}\text { Chabot } \\
\text { (Cottus } \\
\text { gobio) }\end{array}$ & 14 & 70 & 6 & Nég & 6 & Nég & 2 & Nég & & \\
\hline $\begin{array}{l}\text { Epinoche } \\
\text { (Gasterosteus } \\
\text { aculeatus) }\end{array}$ & 1 & 5 & & & 1 & Nég & & & & \\
\hline $\begin{array}{l}\text { Gardon } \\
\text { (Rutilus } \\
\text { rutilus) }\end{array}$ & 7 & 35 & & & 2 & Nèg & & & 5 & Nég \\
\hline $\begin{array}{c}\text { Goujon } \\
\text { (Gobio } \\
\text { gobio) }\end{array}$ & 10 & 50 & 3 & Nèg & 5 & Nég & & & 2 & Nég \\
\hline $\begin{array}{l}\text { Lamproie } \\
\text { (Lampetra } \\
\text { sp.) }\end{array}$ & 1 & 1 & & & 1 & Nég & & & & \\
\hline $\begin{array}{l}\text { Loche } \\
\text { (Cobitis } \\
\text { taenia) }\end{array}$ & 2 & 10 & & & & & 2 & Nég & & \\
\hline $\begin{array}{l}\text { Vairon } \\
\text { (Phoxinus } \\
\text { phoxinus) }\end{array}$ & 26 & 130 & & & & & 23 & Nég & 3 & Nég \\
\hline TOTAL & 85 & 373 & 9 & Nég & 29 & Nég & 27 & Nég & 20 & Nég \\
\hline
\end{tabular}

La sérologie permet de mettre en évidence une contamination virale intervenue dans les mois précédents : le délai de réponse en anticorps étant de 6 semaines minimum et la persistance courte (quelques mois) (HATTENBERGER-BAUDOUY, 1990) (ENZMANN et KONRAD, 1993). Le décalage des prises de sang dans les salmonicultures par rapport à l'étude ne constitue donc pas un problème.

\section{Sérologie des espèces cibles "sauvages"}

Toutes les sérologies $\mathrm{NHI}$ et SHV sont négatives. Plus de $90 \%$ des sérums ont été prélevés en aval d'une ou des deux piscicultures présentant des sérologies positives SHV et/ou NHI lors d'études et où en 1990 l'infection SHV et/ou NHI a été mise en évidence par virologie sans aucun assainissement depuis cette date (figure 1). L'infection présente dans les salmonicultures ne paraît pas entraîner une forte contamination des espèces réceptives dans le milieu naturel.

Cette affirmation doit cependant être nuancée. $80 \%$ des sérums proviennent de truites fario. Par rapport à la truite arc-en-ciel, cette espèce est moins sensible à la SHV, surtout le virus de sérotype 1, et à la NHI (GLASS et al., 1991; LA PATRA et FRYER, 1990). La production d'anticorps est donc moins fréquente. Cependant dans des enquêtes similaires (ENZMANN et KONRAD, 1985 ; KONRAD et al., 1984), des sérologies positives 


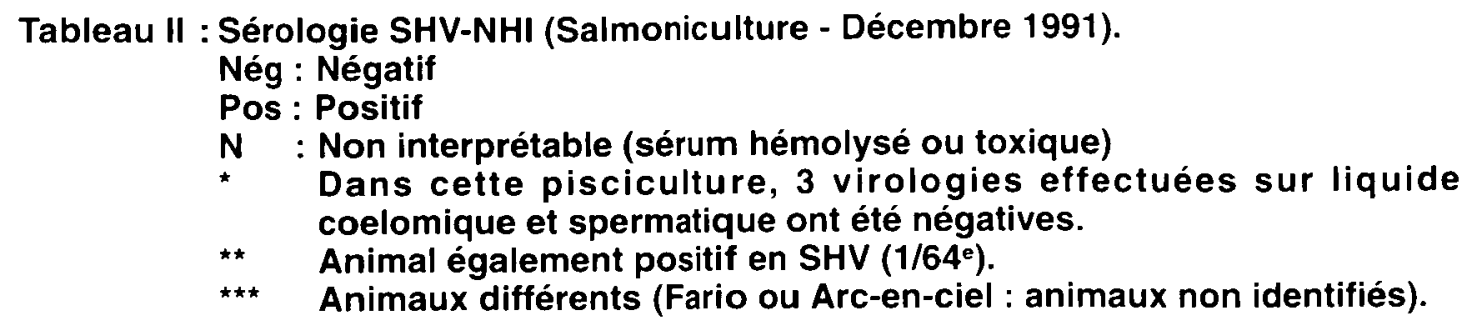

Table II : Serology VHS-IHN (Trout farm - December 1991).

Neg : Negative

Pos : Positive

N : Not interpretable (serum hemolysed or toxic)

* In this trout farm, 3 virological analyses realized on coelomic and spermatic fluid have been negative.

** Animal also positive for VHS $(1 / 64$ e).

$\star \star \star \quad$ Different animals (rainbow or brown trout : animals non identified).

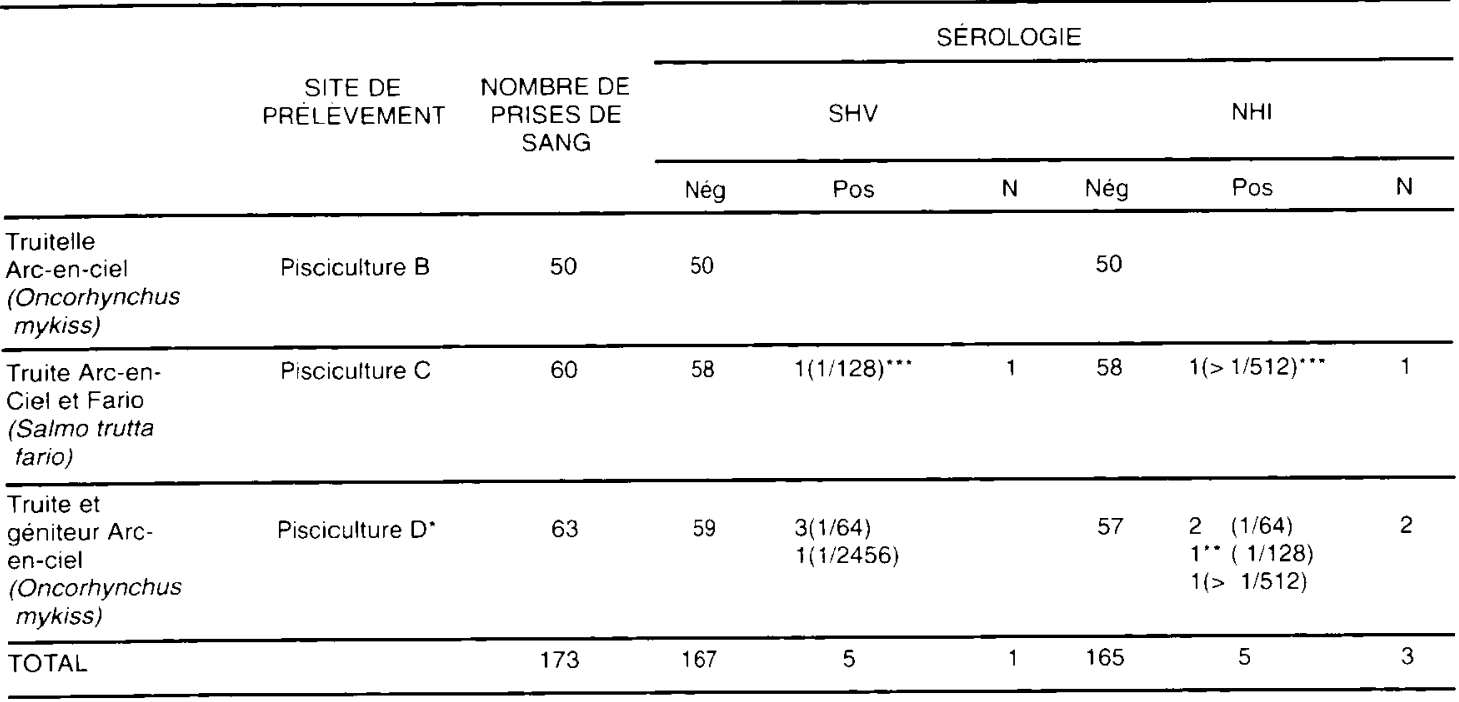

en SHV ont été trouvées sur des truites fario sauvages prélevées dans des cours d'eau en relation avec des salmonicultures où des épisodes cliniques de SHV avaient eu lieu dans les semaines précédentes. Dans notre étude, l'absence de manifestations cliniques de rhabdoviroses, avec pour conséquence une faible pression d'infection sur le milieu naturel, peut expliquer l'absence de sérologies positives chez les truites sauvages aussi bien pour la SHV que pour la NHI, dont le virus a déjà été isolé et des anticorps trouvés chez des truites fario (HATTENBERGER, communication personnelle).

\section{Virologie des espèces autres que Salmonidés}

Plus de $90 \%$ des virologies ont été réalisées à partir de prélèvements effectués en aval d'au moins une pisciculture contaminée (Pisciculture $C$ ou pisciculture $D: 40 \%$ en aval de $\mathrm{C}$ seulement et $50 \%$ en aval des deux). Cependant, le plan d'échantillonnage est peut être insuffisant pour pouvoir conclure à l'absence de portage de rhabdovirus pour les espèces étudiées qui n'ont pas été soumises à une très forte pression d'infection.

L'absence d'épisodes cliniques de rhabdoviroses dans les 4 salmonicultures durant l'étude et la mise en évidence de l'infection seulement par sérologie pour deux d'entre elles laissent à penser que l'excrétion virale par ces piscicultures pendant l'enquête est probablement épisodique et de faible niveau, d'où une faible contamination du milieu, insuffisante pour entretenir un niveau élevé d'infection dans le milieu naturel. 
D'autre part, aucune des espèces étudiées ici n'est considérée comme sensible ou même éventuellement porteuse de deux rhabdovirus, comme cela a été montré pour le brochet qui peut présenter des anticorps contre le virus de la SHV (KONRAD et al., 1984). L'étude du portage viral pourrait être étendue à d'autres vertébrés et invertébrés aquatiques, en particulier à des parasites de salmonidés. En effet, le virus NHI a notamment été isolé de sangsues (Piscicola salmositica) et de copépodes (Salmincola sp.) ectoparasites de saumon (Oncorhynchus nerka) (MULCAHY et al., 1990).

Des études complémentaires, plus longues et plus lourdes, permettraient de mieux cerner l'épidémiologie des rhabdoviroses dans le milieu naturel : utilisation de techniques d'analyse plus sensible, essai de mise en évidence du virus dans le milieu naturel par filtration, enquête similaire menée après un épisode clinique de rhabdovirose, etc....

\section{CONCLUSION}

Cette étude, bien qu'imparfaite, permet cependant de mieux situer le niveau de contamination et de portage de rhabdovirus de différentes espèces sauvages dans un milieu naturel en relation avec des salmonicultures infectées. Au vu des résultats, ce niveau semble relativement bas en l'absence de manifestations cliniques dans les piscicultures avec une incidence dans l'épidémiologie de ces virus probablement faible dans des conditions de pression d'infection virale basse.

\section{REMERCIEMENTS}

Cette étude, réalisée à l'initiative de la Direction des Services Vétérinaires du Pas-deCalais, a été possible grâce à la collaboration efficace de plusieurs organismes et intervenants : le Conseil Supérieur de la Pêche, la Direction des Services Vétérinaires, la Fédération Départementale de Pêche du Pas-de-Calais, le Groupement de Défense Sanitaire Aquacole du Pas-de-Calais, les Pisciculteurs et le Laboratoire Vétérinaire Départemental.

Nous remercions vivement tous les gardes-pêche de la Fédération de Pêche et du Conseil Supérieur de la Pêche qui ont conduit les différentes pêches électriques, ainsi que le Docteur MORAND et Madame HATTENBERGER qui ont bien voulu relire cet article, et Monsieur DORSON qui nous a fourni une importante bibliographie.

\section{BIBLIOGRAPHIE}

AHNE W., THOMSEN I., 1985. Occurrence of VHS in virus in wild white fish (coregonus sp.). Zentralbl. Veterinärmed (B), 32, 73-75.

AMEND D.F., YASUTAKE W.T., MEAD R.W., 1969. A hematopoïetic virus disease of rainbow trout and sockeye salmon. Trans. Am. Fish. Soc., 98, 796.

BELLET R., 1954. Du syndrome entéro-hépato-rénal chez la Truite Arc-en-ciel de pisciculture. Bull. Fr. Piscic., 189, 113 - 124.

BERNARD J., BEARZOTTI - LE BERRE M., DE KINKELIN P., 1985. Viral haemorrhagic septicaemia in rainbow trout : attempt to relate interferon production, antibody synthesis and structure of the virus with the mechanism of virulence. Annales de Virologie (Paris) 136E, 13-26.

DE KINKELIN P., MICHEL Ch., GHITTINO P., 1985. Précis de pathologie des poissons, INRA-OIE.

DE KINKELIN P., HATTENBERGER A.M., TORCHY C., LIEFFRIG F., 1987. Infectious Hematopoietic Necrosis (IHN) : First report in Europe. Third international conference, 31-08 to 3-09-1987, EAFP, Bergen/Norway.

DORSON M., DE KINKELIN P., TORCHY C., MONGE D., 1987. Sensibilité du brochet (Esox lucius) à différents virus de salmonidés (NPI, SHV, NHI) et au rhabdovirus de la perche. Bull. Fr. Pêche Piscic., 307, 91-101. 
ENZMANN P.J., KONRAD M., 1985. Inapparent infections of brown trout with VHS - virus. Bull. Eur. Ass. Fish. Pathol, 5 (4), 81-83.

ENZMANN P.J., KONRAD M., 1993. Longevity of antibodies in brown trout and rainbow trout following experimental infection with VHS - virus. Bull. Eur. Ass. Fish. Pathol., $13(6), 193-194$.

FIJAN N., SULIMANOVIC D., BEARZOTTI M., MUZINIC D., ZWILLENBERG L.O., CHILMONCZYK S., VAUTHEROT J.F., DE KINKELIN P., 1983. Some properties of Epithelioma papulosum cyprini (EPC) cell line from carp Cyprinus carpio. Ann. Virol. (Inst. Pasteur), 134E, 207-220.

GLASS B., KRUSE P., NEUKIRCH M., 1991. Comparative infection studies in brown trout (salmo trutta fario) and rainbow trout (Oncorhynchus mykiss) using several VHS virus type 1 strains. Bull. Eur. Ass. Fish. Pathol., 11 (3), 99-100.

HATTENBERGER - BAUDOUY A.M., DANTON M., MERLE G., TORCHY C., DE KINKELIN P., 1989. Serological evidence of infectious hematopoïetic necrosis in rainbow trout from a French outbreak of disease. Journal of Aquatic Animal Health, 1, 126-134.

HATTENBERGER - BAUDOUY A.M., 1990. Apport de la sérologie dans le diagnostic des maladies virales. Colloque National d'Ichtyopathologie, 20/11/90, EAFP - CNEVALCRV de MAISONS-ALFORT.

JENSEN M.H., 1965. Research on the virus of Egtved disease. Ann. N.Y. Acad. Sci, $126(1), 422-426$.

KONRAD M., RAPP J., ENZMANN P.J., 1984. Epidemiological studies on VHS in a wild fish population. Bull. Eur. Ass. Fish. Pathol., 4 (4), 69-70.

LA PATRA S.E., FRYER J.L., 1990. Susceptibility of brown trout (Salmo trutta) to infections hematopoïetic necrosis virus. Bull. Eur. Ass. Fish. Pathol., 10 (5), 125-127.

MULCAHY D., KLAYBOR D., BATTS W.N., 1990. Isolation of infectious hematopoïetic necrosis virus from a leech (Piscicola salmositica) and a copepod (Salmincola sp.), ectoparasites of sockeye salmon (Oncorhynchus nerka). Dis. aquat. Org., Vol 8, 29-34.

R.N.E. Pr. 109/00/IS 410/00 et IS 290/00, 1993.

IS 290/00 - Détection d'anticorps neutralisant le virus de la nécrose hématopoietique infectieuse dans le sérum des salmonidés par la technique de séroneutralisation en culture cellulaire.

IS 410/00 - Détection d'anticorps neutralisant le virus de la septicémie hémorragique virale dans le sérum des salmonidés par la technique de séroneutralisation en culture cellulaire. Ed. CNEVA de MAISONS/ALFORT.

TACK E., 1959. Beiträge zur Erforschung der Forellensuche Arch. Fischereiwiss, 10, 20-30.

WOLF K., QUIMBY M.C., 1962. Established eurythermic line of fish cell in vitro. Science, 135 (3508), 1065-1066.

ZWILLENBERG L.O., PFITZNER I. and ZWILLENBERG H.H.L., 1968. Infektionsversuche mit Egtved-virus an Zellkulturen und Individuen der Schleie (Tinca vulgaris Cuv) sowie an anderen Fischarten. Zentralbl. Bakteriol. Hyg. I Abt Orig. 208, 218-226. 